\title{
Energy dissipation in the presence of sub-harmonic excitation in dynamic atomic force microscopy
}

\author{
Matteo Chiesa $^{1(a)(b)}$, Karim R. Gadelrab ${ }^{1}$, Albert Verdaguer ${ }^{2}$, J. J. Segura ${ }^{2}$, Victor Barcons ${ }^{3}$, \\ Neil H. Thomson ${ }^{4}$, Michael A. Phillips ${ }^{5}$, Marco Stefancich $^{1}$ and Sergio Santos ${ }^{1(a)}$ \\ ${ }^{1}$ Laboratory of Energy and Nanosciences, Masdar Institute of Science and Technology \\ Abu Dhabi, United Arab Emirates \\ ${ }^{2}$ Centre d'Investigació en Nanociència i Nanotecnologia (CIN2) (CSIC-ICN), Esfera UAB, \\ Campus de la UAB - Edifici CM-7, 08193 Bellaterra, Catalunya, Spain, EU \\ ${ }^{3}$ Departament de Disseny i Programació de Sistemes Electrònics, UPC - Universitat Politècnica de Catalunya \\ Av. Bases, 61, 08242 Manresa, Spain, EU \\ ${ }^{4}$ Department of Oral Biology and School of Physics and Astronomy, University of Leeds - LS2 9JT, Leeds, UK, EU \\ ${ }^{5}$ Asylum Research UK Ltd, Commerce House - Telford Road, Bicester, Oxfordshire OX26 4LD, UK, EU
}

received 1 July 2012; accepted in final form 1 August 2012

published online 11 September 2012

PACS 68.37.Ps - Atomic force microscopy (AFM)

PACS 05.45.-a - Nonlinear dynamics and chaos

\begin{abstract}
Amplitude modulation atomic force microscopy allows quantifying energy dissipation in the nanoscale with great accuracy with the use of analytical expressions that account for the fundamental frequency and higher harmonics. Here, we focus on the effects of sub-harmonic excitation on energy dissipation and its quantification. While there might be several mechanisms inducing sub-harmonics, a general analytical expression to quantify energy dissipation whenever sub-harmonics are excited is provided. The expression is a generalization of previous findings. We validate the expression via numerical integration by considering capillary forces and provide experimental evidence of sub-harmonic excitation for a range of operational parameters.
\end{abstract}

Copyright (c) EPLA, 2012

Dynamic atomic force microscopy has been widely employed to investigate energy dissipation in the nanoscale [1-8]. In particular, the theory of amplitude modulation (AM) AFM can be briefly summarized as the study of the behavior of the phase $\varphi$ of the fundamental frequency relative to the phase of the driving force. The study of energy dissipation is providing new insights into the mechanisms responsible for a variety of phenomena from the heterogeneity of viscoelasticity in metallic glasses [9] to the implications of energy dissipation and nanoscale heterogeneity in bones [10] and nanoscale capillary interactions [3]. It is expected however that, with further developments, the applications of these methods will increase and broaden in scope.

It has been long established that $\varphi$ varies depending on 1) how much energy is being dissipated in the interaction (dissipative component) [4] and 2) the perturbed amplitude $A_{1}$ of oscillation of the fundamental frequency

\footnotetext{
(a) These authors contributed equally to this paper.

(b) E-mail: mchiesa@masdar . ac . ae
}

(conservative component) $[4,11,12]$. In ambient conditions, where the $Q$ factor is large, i.e., $\sim 10^{2}-10^{3}$, the amplitudes of the higher harmonics can be neglected since their contribution to the mean energy dissipated per cycle $\left\langle E_{\text {dis }}\right\rangle$ is less than $1 \%[4,12]$. Then, by applying the energy conservation principle for one oscillation cycle, it is found from the fundamental frequency $f$ alone that [4]

$$
\left\langle E_{\mathrm{dis}}\right\rangle=\frac{\pi k A_{0} A_{1}}{Q}\left[\sin \varphi-\frac{A_{1}}{A_{0}}\right]
$$

where $A_{0}$ is the free amplitude of oscillation, $k$ is the spring constant and $Q$ is the $Q$ factor due to dissipation with the medium. Note that while (1) is only valid when driving at the natural frequency of oscillation $\omega=\omega_{0}$, a simple modification leads to the general solution for any drive frequency $\omega[13] ; \omega$ and $\omega_{0}$ are the drive and natural angular frequencies, respectively. Equation (1) has been widely used to identify [1] and quantify [3,11,14,15] energy dissipation processes, and, in particular, dissipation processes where capillary interactions are involved [3,14,15]. Still, in one of the first studies of capillary interactions in 
dynamic AFM, Zitzler et al. already reported that (1) might not predict $\left\langle E_{\text {dis }}\right\rangle$ accurately in certain situations. In their study, higher harmonics were thought to account for the discrepancy between (1) and the expected $\left\langle E_{\text {dis }}\right\rangle$ values [14]. For completeness, and for later comparison of results, the expression accounting for the energy dissipated via higher harmonics as derived by Tamayo [12] is also given here,

$$
\left\langle E_{\mathrm{dis}}\right\rangle=\frac{\pi k A_{0} A_{1}}{Q}\left[\sin \varphi-\frac{1}{A_{0} A_{1}} \sum_{n \geqslant 1} n^{2} A_{n}^{2}\right] .
$$

It should be noted that significant higher harmonic amplitudes $A_{n}$ are characteristic of liquid imaging where the $Q$ factor is 10 or less. Thus, it is not surprising that, in ambient conditions, (1) alone is considered [1,3,12].

Here, we report that in ambient conditions, i.e., $Q \sim$ $10^{2}-10^{3}$, and, as already reported by Tamayo, (1) and (2) are practically equivalent with predictions producing differences of less than 1\%. Furthermore, we report that the main assumption in the derivation of (1) and (2), namely, the application of the energy conservation principle during one fundamental oscillation cycle, does not apply when sub-harmonics are excited. In general, when sub-harmonics are present, the energy conservation principle should be applied for $p$ fundamental oscillation cycles, where $p$ is the ratio between the fundamental frequency $\omega$ or $f$ and the sub-harmonic fundamental frequency $\omega_{\mathrm{SH}}$ or $f_{\mathrm{SH}}$. That is, $p=f / f_{\mathrm{SH}}$. Harmonics, i.e., $n f_{\mathrm{SH}}$, of $f_{\mathrm{SH}}$ might also be present in the signal. Sub-harmonic $(\mathrm{SH})$ excitation might occur, for example, in the presence of hysteretic forces where the onset of the force occurs on tip approach at a tip-sample distance $d_{\mathrm{on}}$ and the breakoff occurs on tip retraction at a distance $d_{\text {off }}$ and where $d_{\text {off }}>d_{\text {on }}[16,17]$. A common example where this occurs is when the capillary force is present $[14-18]$. An example of the type of tip-sample force $F_{\mathrm{ts}}$ dependencies on tip-sample distance $d$ which might arise in the presence of capillary interactions is given in fig. 1(a). The long range hysteresis shown in the figure leads to energy dissipation (colored area in fig. $1(\mathrm{a}))$ where a $d_{\text {off }} / d_{\text {on }}$ mechanism controls the interaction. The details on the forces are given below. Recently, it has been shown that the stochastic formation and rupture of atomic chains in the tipsample junction can lead to significant dissipation in the interaction [6]. Since such processes occur with frequencies lower than the fundamental and might involve a $d_{\text {off }} / d_{\text {on }}$ hysteretic mechanism, these phenomena should also excite sub-harmonics even though it is not intended to pursue such study here. In summary, in this work a general expression for the energy dissipated in the presence of SHs is derived from the energy conservation principle. The conditions for which SHs are excited in the presence of hysteretic forces that have $d_{\text {off }} / d_{\text {on }}$ mechanisms, in particular for the capillary force, are also elucidated. The theory is validated via numerical methods and experimental evidence of $\mathrm{SH}$ excitation under standard conditions is

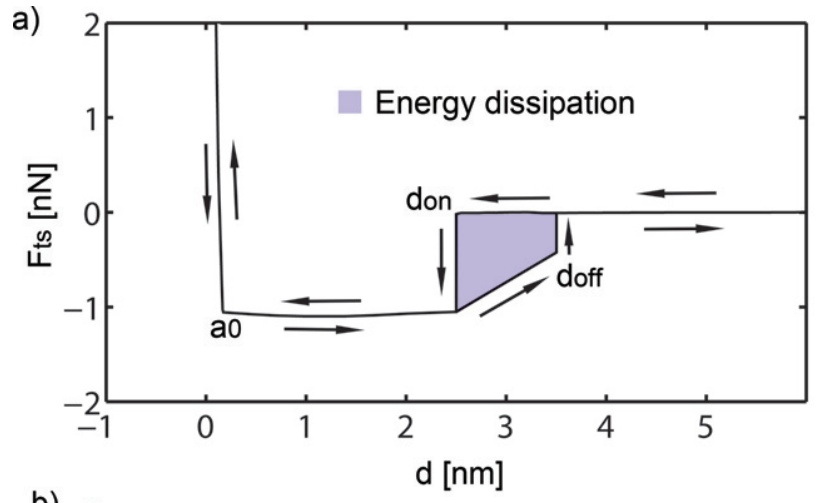

b)

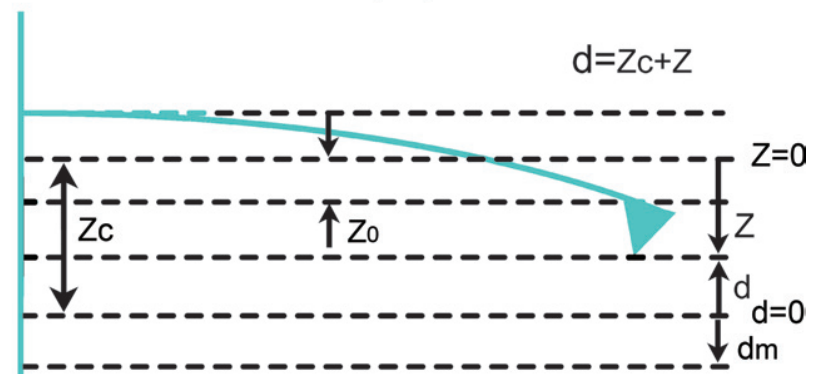

Fig. 1: (Color online) (a) Form for the net tip-sample $F_{\mathrm{ts}}$ force $v s$. tip-sample distance $d$ used in this work for numerical integration [17]; $a_{0}$ is an intermolecular distance. A form of hysteresis is present where energy is dissipated (colored area) due to differences in the onset $\left(d_{\mathrm{on}}\right)$ and breakoff distance $\left(d_{\mathrm{off}}\right)$ of the capillary force [17]. (b) Scheme of a cantilever vibrating over a surface where the instantaneous tip position $z$ relative to unperturbed equilibrium position of the cantilever, $d$, mean cantilever deflection $z_{0}$, the cantilever-sample separation $z_{\mathrm{c}}$ and minimum distance of approach $d_{\mathrm{m}}$ are shown. Both $d$ and $z$ are defined as positive in the upward direction.

provided. The expressions (1) and (2) are compared to the more general expression derived in this work in both simulations and experiment and shown to be valid provided SHs are not excited.

Let us first consider an expression for the net tipsample force $F_{\text {ts }}$ where the capillary interaction is present (fig. 1(a)). A scheme of the cantilever-sample geometry is given in fig. 1(b). Note the geometrical relationship $d=$ $z_{\mathrm{c}}+z$ where $d$ is the instantaneous tip-sample distance, $z_{\mathrm{c}}$ is the cantilever-sample separation and $z$ is the tip position. This relationship is typically used in the literature when modeling AM AFM $[19,20]$. The minimum distance of approach $d_{\mathrm{m}}$ is also shown in the figure and might be positive or negative. Both $z$ and $d$ are defined positive in the upward vertical direction. The equation of motion in ambient dynamic AFM can be approximated, to its first mode [19], to

$$
m \frac{\mathrm{d}^{2} z}{\mathrm{~d} t^{2}}+\frac{m \omega}{Q} \frac{\mathrm{d} z}{\mathrm{~d} t}+k z=F_{\mathrm{ts}}+F_{0} \cos \omega t,
$$

where $m=k / \omega^{2}$ and $F_{0}$ is the amplitude of the driving force. A net tip-sample force $F_{\mathrm{ts}}$ that closely reproduces standard phenomena in ambient AM AFM in the presence 
of water films (fig. 1(a)) can be written in terms of $d[16,17]$. Next, such model is described in order to establish, with the use of simulations, whether (1) and (2) hold when SHs are excited. In the long range, the attractive London dispersion force $F_{\mathrm{a}}$ is

$$
\begin{aligned}
& F_{\mathrm{a}}(d)=-\frac{H_{\mathrm{w}} R}{6 d_{\mathrm{w}}^{2}}, \\
& d>d_{\mathrm{off}}\left(\text { tip retraction) and } d>d_{\mathrm{on}}(\text { tip approach), }\right.
\end{aligned}
$$

where $H_{\mathrm{w}}$ is the Hamaker constant corresponding to the hydrated tip-hydrated surface interaction, $R$ is the tip radius and $d_{\mathrm{w}}$ is an effective distance. Here, $d_{\mathrm{w}}$ takes into account that in the presence of adsorbed water films $[16,17,21]$ the effective distance is $d_{\mathrm{w}}=d-2 h$ where $h$ is the height of the water films on the tip and sample's surfaces. In the metastable region, i.e., $d_{\mathrm{off}}>d>d_{\mathrm{on}}$, the capillary neck might be formed. In this case (4) can be identified with the adhesion force $F_{\mathrm{AD}}[17]$

$$
F_{\mathrm{AD}}(d)=\frac{F_{d_{\mathrm{off}}}-F_{d_{\mathrm{on}}}}{d_{\mathrm{off}}-d_{\mathrm{on}}}\left(d-d_{\mathrm{on}}\right)+F_{d_{\mathrm{on}}}, \quad d_{\mathrm{off}}>d>d_{\mathrm{on}},
$$

where

$$
F_{d_{\mathrm{on}}}=-\frac{R}{6 a_{0}^{2}}\left[\frac{H_{\mathrm{w}}-H}{d_{\mathrm{off}}-a_{0}}\left(d_{\mathrm{on}}-a_{0}\right)+H\right]
$$

and

$$
F_{d_{\text {off }}}=-C_{\text {off }} \frac{R H_{\mathrm{w}}}{6 a_{0}^{2}}, \quad C_{\text {off }} \geqslant 0 .
$$

$H$ is the Hamaker constant for the tip and the sample interaction, $a_{0}$ is an intermolecular distance that implies that matter interpenetration cannot occur and $C_{\text {off }}$ controls the decay in the adhesion force for $d>d_{\text {on }}$. Here $C_{\text {off }}=0.3$ has been used here throughout. In the region $a_{0}<d<d_{\text {on }}$ the adhesion force due to London dispersion forces is written as $[17,21]$

$$
F_{\mathrm{AD}}(d)=-\frac{H^{*} R}{6 a_{0}^{2}}, \quad a_{0}<d<d_{\mathrm{on}},
$$

where

$$
H^{*}=\frac{H_{\mathrm{w}}-H}{d_{\mathrm{off}}-a_{0}}\left(d-a_{0}\right)+H .
$$

Finally, when mechanical contact occurs, $d \leqslant a_{0}$, the adhesion force is

$$
F_{\mathrm{AD}}=-\frac{H R}{6 a_{0}^{2}}=-4 \pi R \gamma, \quad d \leqslant a_{0},
$$

where $\gamma$ is the surface energy. When the capillary neck is formed the capillary force is $[17,22]$

$F_{\mathrm{CAP}}(d)=-\frac{2 \pi \gamma_{\mathrm{w}} R}{1+\frac{\pi d^{3}}{V_{\mathrm{men}}}}, \quad$ provided capillary on and $d>a_{0}$,

where $\gamma_{\mathrm{w}}$ is the surface energy of water and $V_{\text {men }}$ is the volume of the meniscus or the volume of water forming the water bridge. Note that $H_{\mathrm{w}} \approx 24 \pi\left(a_{0}\right)^{2} \gamma_{\mathrm{w}}$ and $H \approx$ $24 \pi\left(a_{0}\right)^{2} \gamma[23]$. When $d \leqslant a_{0}$, the distance $d$ is replaced by $a_{0}$ in (11). In (11) $V_{\text {men }}$ is calculated using geometrical considerations [14]

$$
V_{\text {men }}=4 \pi R h^{2}+\frac{4}{3} \pi h^{3} .
$$

The distance $d_{\text {on }}$ has been taken to be $d_{\text {on }}=3 h$ [17]. Additionally $d_{\text {off }}$ can be calculated numerically by solving the Laplace-Young equation to give $[14,24]$

$$
d_{\mathrm{off}} \approx V_{\mathrm{men}}^{1 / 3}-\frac{1}{5 R} V_{\mathrm{men}}^{2 / 3} .
$$

Finally, when contact occurs, i.e., $d \leqslant a_{0}$, the conservative short range repulsive force is written, from the Derjaguin-Muller-Toporov (DMT) model [25]

$$
F_{\mathrm{DMT}}(d)=\frac{4}{3} E^{*} \sqrt{R} \delta^{3 / 2}, \quad d \leqslant a_{0}
$$

where $E^{*}$ is the effective elastic modulus of the contacting bodies and $\delta$ is the sample deformation, i.e., $\delta=a_{0}-$ $d[26]$. The implementation of (4) to (14) results in a tipsample force $F_{\mathrm{ts}} v s$. distance $d$ as shown in fig. 1(a). The parameters are $E_{\mathrm{t}}=170 \mathrm{GPa}$ (Young modulus of the tip), $E_{\mathrm{s}}=60 \mathrm{GPa}$ (Young modulus of the sample), $R=7 \mathrm{~nm}$, $h=0.8 \mathrm{~nm}, \gamma=7 \mathrm{~mJ}, \gamma_{\mathrm{w}}=10 \mathrm{~mJ}$ and $a_{0}=0.165 \mathrm{~nm}$ [17]. The colored area in fig. 1(a) coincides with the energy dissipated due to capillary neck formation and rupture. In the example in fig. $1(\mathrm{~b})\left\langle E_{\text {dis }}\right\rangle \approx 4.6 \mathrm{eV}$ follows. Since no other mechanism for energy dissipation is accounted for in this model, the possibilities are: 1) the capillary neck forms and ruptures during each cycle dissipating $\left\langle E_{\mathrm{dis}}\right\rangle=4.6 \mathrm{eV}$, or 2) the capillary does not form nor rupture during one cycle thus dissipating $\left\langle E_{\text {dis }}\right\rangle=0 \mathrm{eV}$. Next, the equation of motion (3) is solved numerically in order to establish the validity of (1) and (2).

Here, (3) has been implemented both in Matlab and $\mathrm{C}$ and standard Runge-Kutta and Adams-Bashforth algorithms of the fourth order have been employed to check the robustness of the methods. No difference in the outcome has been observed (data not shown). The cantilever parameters are $k=2 \mathrm{~N} / \mathrm{m}, Q=120$ and $\omega=2 \pi f_{0} \quad\left(f_{0}=\right.$ $70 \mathrm{kHz}$ ). In figs. $2(\mathrm{a}),(\mathrm{b}), A_{0}=1 \mathrm{~nm}$ and $z_{\mathrm{c}}=3.5 \mathrm{~nm}$. This produced a set-point amplitude $A_{1} \approx 0.9 \mathrm{~nm}$. These conditions, i.e., small values of $A_{0}$ and high values of $A_{1}$, are known to lead to a pure non-contact mode of operation $[16,21]$. This is in fact observed by looking at the wave form in fig. 2(b) where the tip always oscillates above the distance $d_{\text {on }}\left(d_{\text {on }}=3 h=2.4 \mathrm{~nm}\right)$. In fig. 2(a) an FFT of the wave form shows that no $\mathrm{SH}$ s are excited. The amplitudes of the higher harmonics here lie below $1 \mathrm{pm}$ (data not shown). From (1) and (2) (for $N=100$ harmonics) $\left\langle E_{\text {dis }}\right\rangle \approx 0.0 \mathrm{eV}$ is obtained in this case (figs. 2(a), (b)) agreeing with the expected dissipation.

Increasing the free amplitude $A_{0}$ results in $\mathrm{SH}$ excitation. This can be observed in figs. 2(c), (d) where 

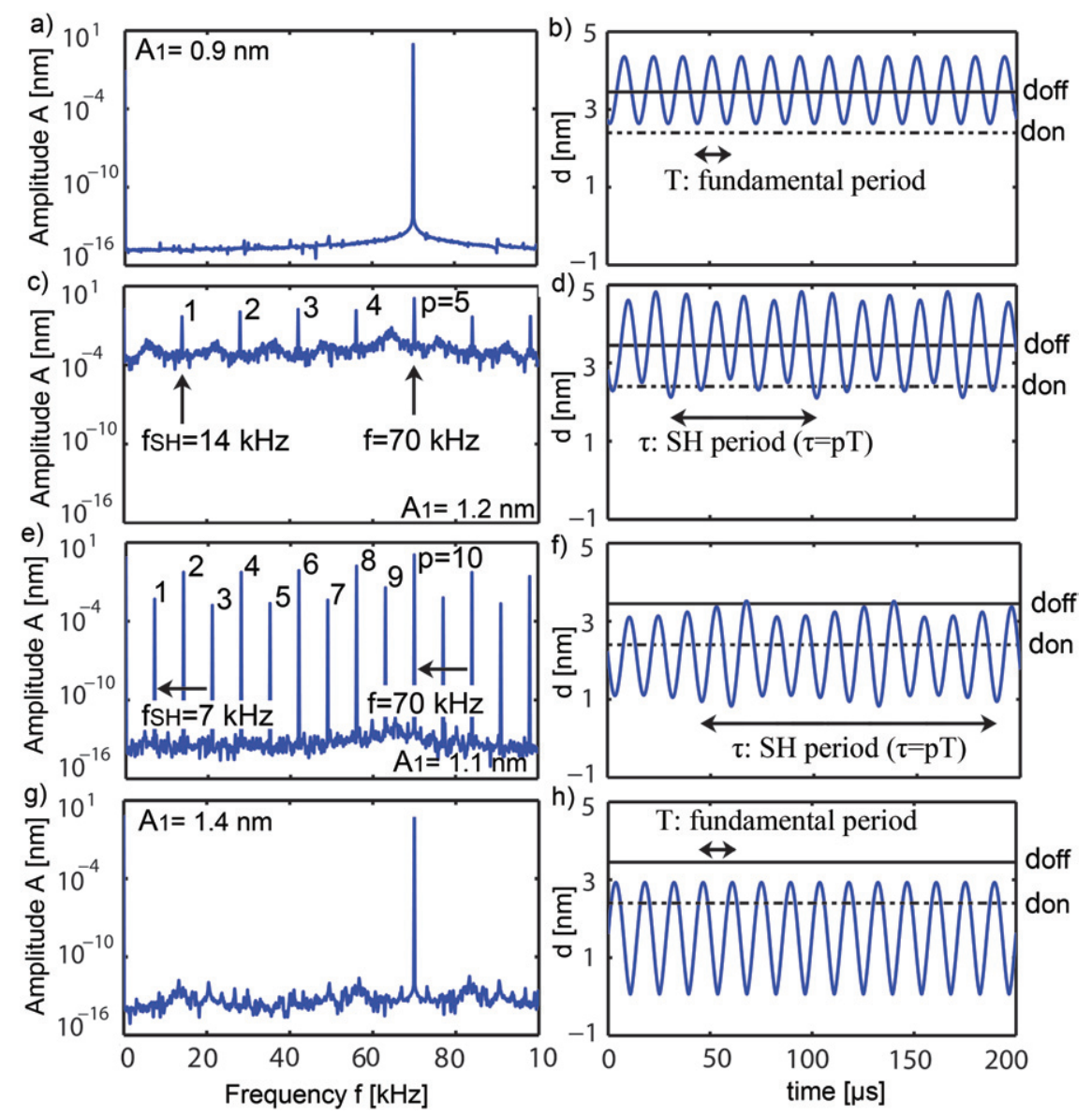

Fig. 2: (Color online) Numerical integration of the equation of motion shows that (a) sub-harmonics are not excited (FFT left column and wave form right column) if the tip oscillates high enough above the sample so that the minimum distance of approach $d_{\mathrm{m}}$ is always larger than $d_{\mathrm{on}}$. Here $A_{0}=1 \mathrm{~nm}$. In (c) and (e) the capillary breaks and ruptures and the motion of the tip becomes periodic with period $\tau$ this being larger than the fundamental period $T$, i.e., $\tau=p T$ where $p$ is an integer. (g) If the tip always oscillates below the distance $d_{\text {off }}$, the capillary never ruptures and sub-harmonics are not excited due to capillary interactions. From (c) to (h) $A_{0}=20 \mathrm{~nm}$.

$A_{0}=20 \mathrm{~nm}, z_{\mathrm{c}}=3.6 \mathrm{~nm}$ and $A_{1}=1.2 \mathrm{~nm}$. Under these conditions (fig. 2(c)) $f_{\mathrm{SH}}=14 \mathrm{kHz}$. Thus, $p=f / f_{\mathrm{SH}}=5$ $(f=70 \mathrm{kHz})$. Here, $\mathrm{SH}$ excitation follows from the loss of energy caused by intermittent water impacts with $\mathrm{SH}$ period $\tau$ [16]. More thoroughly, the period in fig. 2(d) is $\tau=1 / f_{\mathrm{SH}}$ or $\tau=p 1 / f=p T$ where $\tau$ is the SH period, $T$ is the fundamental period, $p=5$ and $d_{\mathrm{on}}$ is reached during 3 out of 5 fundamental cycles only, that is the capillary forms and ruptures during 3 out of 5 fundamental cycles or $3 / 5$. In this case both (1) and $(2)(N=100)$ predict $\left\langle E_{\mathrm{dis}}\right\rangle \approx 2.8 \mathrm{eV}$. That is, $4.6 \mathrm{eV}$ is not recovered even when considering the higher harmonics. By closer inspection, however, (1) and (2) approximately recover the mean energy dissipated during every $p$ fundamental periods $T$ or single period $\tau$ instead. For example, $4.6 \mathrm{eV}$ can be recovered from (1) and (2) if one knows the number of cycles for which, during a $\mathrm{SH}$ period $\tau$, the capillary ruptures and forms. Experimentally this is unknown. However, from fig. 2 (d) the reciprocal ratio is $5 / 3$. Then $\left\langle E_{\text {dis }}\right\rangle \approx 2.8 \mathrm{eV} \times$ $\frac{5}{3} \approx 4.6 \mathrm{eV}$ (for $N=1$ and $p=5$ in (1) and/or (2)). In figs. 2(e), (f) the separation $z_{\mathrm{c}}$ has been reduced to $2.6 \mathrm{~nm}$. This results in $A_{1}=1.1 \mathrm{~nm}$ and, again, the excitation of SHs. The SH period here is $\tau=10 T$. However, in this case the tip oscillates inside the capillary neck during most of the $\mathrm{SH}$ period $\tau$. Note that during each fundamental period $T$ the condition $d_{\mathrm{m}}<d_{\text {on }}$ is always satisfied. The mechanism for SH excitation here is thus slightly different. Now, both (1) and (2) $(N=100)$ predict $\left\langle E_{\text {dis }}\right\rangle=0.9 \mathrm{eV}$. Again, from the ratio 10/2 observed in fig. $2(\mathrm{f}),\left\langle E_{\text {dis }}\right\rangle=$ $0.9 \mathrm{eV} \times \frac{10}{2} \approx 4.6 \mathrm{eV}$ is recovered. According to our simulations the cases illustrated in figs. 2(c), (d) and 2(e), (f) are the two scenarios where $\mathrm{SH}$ excitation is induced by forces presenting $d_{\text {off }} / d_{\text {on }}$ mechanisms. Another possibility is illustrated in figs. $2(\mathrm{~g})$, (h) where $d<d_{\text {off }}$ throughout and where $z_{\mathrm{c}}=2 \mathrm{~nm}$ and $A_{1}=1.4 \mathrm{~nm}$. This case has been recently reported and consists of a perpetual water contact mode. Note that even though $z_{\mathrm{c}}$ is now smaller, $A_{1}$ is larger [16,17]. No SHs are excited (fig. 2(g)) and the periodicity $\tau=T$ is recovered (fig. 2(h)). As expected, both (1) and (2) $(N=100)$ predict $\left\langle E_{\text {dis }}\right\rangle=0.0 \mathrm{eV}$. 
The derivations of (1) and (2) assumed [4,12] that the energy entering the cantilever per cycle $\left\langle E_{\text {in }}\right\rangle$ is the sum of the energy dissipated to the medium $\left\langle E_{\mathrm{m}}\right\rangle$ and the energy dissipated in the tip-sample interaction $\left\langle E_{\mathrm{dis}}\right\rangle$ during a fundamental oscillation period $T$, i.e., $\left\langle E_{\text {in }}\right\rangle=$ $\left\langle E_{\mathrm{m}}\right\rangle+\left\langle E_{\mathrm{dis}}\right\rangle$ for $t^{\prime}<t<t^{\prime}+T$ where $t$ stands for time and $t^{\prime}$ is just a reference. Since in the presence of SHs this condition is not met, an alternative derivation should be carried out. Let us assume that the $\mathrm{SH}$ period is $\tau$ where $\tau=p T$. Then, $\left\langle E_{\text {in }}\right\rangle=\left\langle E_{\mathrm{m}}\right\rangle+\left\langle E_{\text {dis }}\right\rangle$ applies for $t^{\prime}<t<t^{\prime}+\tau$. The position of the tip can be written as (fig. 1(b))

$$
z=z_{0}+\sum_{n \geqslant 1} A_{n} \cos \left(n \omega_{\mathrm{SH}} t-\varphi_{n}\right)
$$

Then, from (3) and for $\omega=\omega_{0}=p \omega_{\mathrm{SH}}$ and integrating over the $\mathrm{SH}$ period $\tau$

$$
\begin{aligned}
\left\langle E_{\text {in }}\right\rangle_{\tau}= & F_{0} \int_{t=t^{\prime}}^{t=t^{\prime}+\tau} \cos p \omega_{\mathrm{SH}} t\left[-\sum_{n \geqslant 1} A_{n} n \omega_{\mathrm{SH}}\right. \\
& \left.\times \sin \left(n \omega_{\mathrm{SH}} t-\varphi_{n}\right)\right] \mathrm{d} t=p \frac{k \pi A_{0} A_{1}}{Q} \sin \varphi_{\mathrm{p}},
\end{aligned}
$$

where $\varphi_{\mathrm{p}}$ coincides with the phase lag of the fundamental frequency, i.e., $f_{\mathrm{p}}=f$, relative to the drive. That is, $\varphi_{\mathrm{p}}$ is simply $\varphi$, i.e., the phase given by standard lockin amplifiers in AFM. The energy dissipated with the medium is

$$
\begin{aligned}
& \left\langle E_{\mathrm{m}}\right\rangle_{\tau}=\frac{k}{Q \omega_{0}} \int_{t=t^{\prime}}^{t=t^{\prime}+\tau} \sum_{n \geqslant 1} \sum_{m \geqslant 1} A_{n} A_{m} n m \omega_{\mathrm{SH}}^{2} \\
& \times \sin \left(n \omega_{\mathrm{SH}} t-\varphi_{n}\right) \sin \left(m \omega_{\mathrm{SH}} t-\varphi_{m}\right) \mathrm{d} t= \\
& \frac{\pi k}{Q} \frac{1}{p} \sum_{n \geqslant 1} n^{2} A_{n}^{2} .
\end{aligned}
$$

Finally

$$
\left\langle E_{\mathrm{dis}}\right\rangle_{\tau}=\frac{\pi k A_{0} A_{1}}{Q}\left[p \sin \varphi-\frac{1}{A_{0} A_{1}} \frac{1}{p} \sum_{n \geqslant 1} n^{2} A_{n}^{2}\right] .
$$

The above expression (18) is a more general form of (1) and (2), takes into account the amplitude of the SHs and implies that energy is conserved during the SH period $\tau$. If $p=1$, (18) reduces to (2). For the conditions in figs. 2(c), (d), (18) gives $\left\langle E_{\mathrm{dis}}\right\rangle=13.9 \mathrm{eV}(N=5)$ agreeing with the fact that during the SH period $\tau=5 T(p=5)$ the capillary forms and ruptures 3 times. For the conditions in figs. 2(e), (f), (18) gives $\left\langle E_{\mathrm{dis}}\right\rangle=9.3 \mathrm{eV}(N=10)$ again agreeing with the fact that during the SH period $\tau=10 T(p=10)$ and the capillary forms and ruptures twice.

Experimental verification of SHs occurring in ambient conditions is provided in fig. 3 on a freshly cleaved mica sample at $50 \%$ relative humidity and with a cantilever

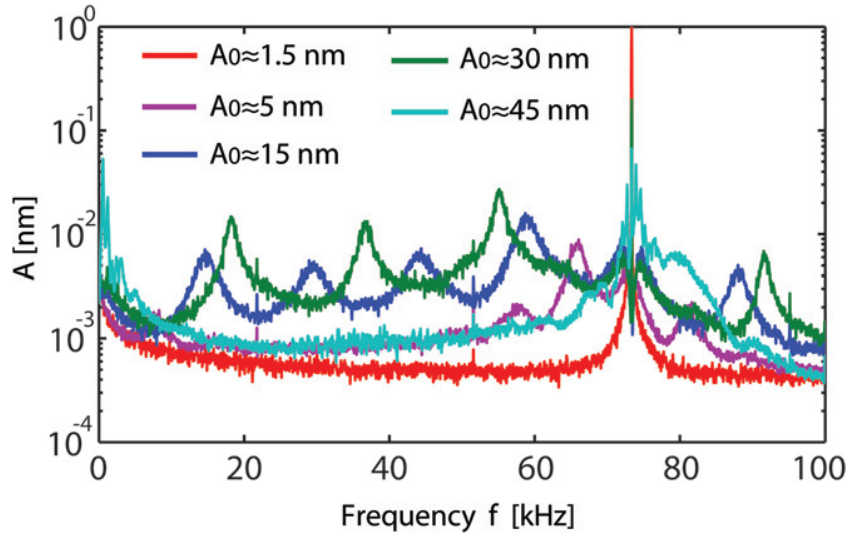

Fig. 3: (Color online) Experimental (FFT) observation of sub-harmonic excitation on a freshly cleaved mica sample at $50 \%$ relative humidity. The set-point was set to $A_{1}=1 \mathrm{~nm}$ throughout and, as the free amplitude is varied from $A_{0}=$ $1.5 \mathrm{~nm}$ to $45 \mathrm{~nm}$ (where the repulsive regime prevails, data not shown), the minimum distance of approach decreases. Subharmonics are excited mainly in the region $5<A_{0}<30 \mathrm{~nm}$.

(OLYMPUS AC240TS) with parameters $k \approx 2 \mathrm{~N}, Q \approx 120$, $f=f_{0} \approx 70 \mathrm{kHz}$ and $R \approx 10 \mathrm{~nm}$. The tip radius has been characterized by monitoring the transition from the attractive to the repulsive regime in situ [27] and $k, Q$ and $f_{0}$ have been calibrated using a standard thermal analysis on a Cypher AFM from Asylum Research [28]. In the experiments, the drive frequency $f$ is constant and set equal to the natural resonant frequency $f_{0}$. This is in accordance with the derivations of (1), (2) and (18). Furthermore, $f_{0}$ is obtained for the free oscillating cantilever, i.e., when the cantilever is oscillating far enough from the surface $z_{\mathrm{c}} \gg A_{0}$, and it is customary in AM AFM [4,11]. The set-point amplitude was set to $A_{1} \approx 1 \mathrm{~nm}$ in the experiment and an FFT was obtained for $A_{0} \approx 1.5 \mathrm{~nm}$ (red lines), $A_{0} \approx 5 \mathrm{~nm}$ (purple lines), $A_{0} \approx 15 \mathrm{~nm}$ (blue lines), $A_{0} \approx 30 \mathrm{~nm}$ (green lines) and $A_{0} \approx 45 \mathrm{~nm}$ (purple lines). The FFTs were acquired with the standard thermal analysis of the Cypher while tapping. It is well known that for a given set-point $A_{1}$ the minimum distance of approach $d_{\mathrm{m}}$ decreases with increases free amplitude $A_{0}[1,13,16]$. Thus, increasing $A_{0}$ while maintaining $A_{1}$ constant provides an experimental method to explore the behavior of the cantilever with decreasing $d_{\mathrm{m}}$. In the figure, for $A_{0}=30 \mathrm{~nm}$ (green lines), the amplitudes of the SHs can reach up to fractions of an angstrom. Note that when $A_{0}=15 \mathrm{~nm}, \tau=5 T$ and when $A_{0}=30 \mathrm{~nm}, \tau=4 T$. For these two values of $A_{0} \quad(1)$ gives $\left\langle E_{\mathrm{dis}}\right\rangle=1.2 \pm 0.2 \mathrm{eV} \quad\left(\varphi=162 \pm 2^{\circ}\right)$ and $\left\langle E_{\mathrm{dis}}\right\rangle=1.7 \pm 0.3 \mathrm{eV} \quad\left(\varphi=168 \pm 2^{\circ}\right)$, respectively. Taking the first $p$ sub-harmonics into account (18) gives $\left\langle E_{\mathrm{dis}}\right\rangle_{\tau}=6 \pm 0.8 \mathrm{eV}(p=5)$ for $\varphi=162 \pm 2^{\circ}$ (blue lines $)$, and $\left\langle E_{\mathrm{dis}}\right\rangle_{\tau}=6.8 \pm 1.2 \mathrm{eV}(p=4)$ for $\varphi=168 \pm 2^{\circ}$ (green lines). The excitation of sub-harmonics under the conditions in fig. 3 could be due to capillary interactions since the behavior agrees with recently reported expected 
conditions. Nevertheless, further experimentation and modeling is required to establish the true source of excitation. Finally, while an FFT will produce a single DC value $z_{0}$ (fig. $1(\mathrm{~b})$ ) and the corresponding frequency contributions (see (15)), it could be argued that the mean deflection $z_{0}$ could significantly vary during a single fundamental $\mathrm{SH}$ period. Such phenomena might be used together with recent advances in dynamic AFM [29] theory to directly calculate the force and frequency shift produced by $d_{\text {off }} / d_{\text {on }}$ mechanisms involved with $\mathrm{SH}$ excitation.

In summary, we have shown that sub-harmonic excitation is predicted to occur in the presence of hysteretic forces where the onset and breakoff of the force takes place at different distances. When sub-harmonics are excited, standard energy dissipation expressions provide the mean value of the energy dissipated over the fundamental subharmonic period. A general close form expression that accounts for sub-harmonic excitation has been provided and shown to agree with numerical results. The excitation of sub-harmonics and the expression for energy dissipation provided in this work could lead to rapid quantification of the processes that cause them, such as possible capillary neck formation and rupture [16] or other [6], with nanoscale resolution.

\section{REFERENCES}

[1] Garcia R., Gómez C. J., Martinez N. F., Patil S., Dietz C. and Magerle R., Phys. Rev. Lett., 97 (2006) 016103.

[2] Garcia R., Magerele R. and Perez R., Nat. Mater., 6 (2007) 405.

[3] Sahagun E., Garcia-Mochales P., Sacha G. M. and Saenz J. J., Phys. Rev. Lett., 98 (2007) 176106.

[4] Cleveland J. P., Anczykowski B., Schmid A. E. and Elings V. B., Appl. Phys. Lett., 72 (1998) 2613.

[5] Hoffmann P. M., Jeffere S., Pethica J. B., Özer H. Ö. and Oral A., Phys. Rev. Lett., 87 (2001) 265502.

[6] Kawai S., Federici Canova F., Glatzel T., Foster A. S. and Meyer E., Phys. Rev. B, 84 (2011) 115415.

[7] Santos S., Gadelrab K. R., Silvernail A., Armstrong P., Stefancich M. and Chiesa M., Nanotechnology, 23 (2012) 125401.
[8] Payam A. F., Ramos J. R. and Garcia R., ACS Nano, 6 (2012) 4663.

[9] Liu Y. H., Wang D., Nakajima K., Zhang W., Hirata A., Nishi T., Inoue A. and Chen M. W., Phys. Rev. Lett., 106 (2011) 125504.

[10] Tai K., DaO M., Suresh S., Palazoglu A. and Ortiz C., Nat. Mater., 6 (2007) 454.

[11] Martinez N. and Garcia R., Nanotechnology, 17 (2006) S167.

[12] Tamayo J., Appl. Phys. Lett., 75 (1999) 3569.

[13] HU S. and RAman A., Nanotechnology, 19 (2008) 375704 .

[14] Zitzler L., Herminghaus S. and Mugele F., Phys. Rev. B, 66 (2002) 155436.

[15] Korayem M. H., Eghbal M. M. and Ebrahimi N., J. Appl. Phys., 110 (2011) 024512.

[16] Santos S., Barcons V., Verdaguer A. and Chiesa M., J. Appl. Phys., 110 (2011) 114902.

[17] Barcons V., Verdaguer A., Font J., Chiesa M. and Santos S., J. Phys. Chem. C, 116 (2012) 7757.

[18] Chiesa M., Gadelrab K., Stefancich M., Armstrong P., Li G., Souier T., Thomson N. H., Barcons V., Font J., Verdaguer A., Phillips M. A. and Santos S., J. Phys. Chem. Lett., 3 (2012) 2125.

[19] Garcia R. and San Paulo A., Phys. Rev. B, 60 (1999) 4961.

[20] Santos S., Gadelrab K., Barcons V., Stefancich M. and Chiesa M., New J. Phys., 14 (2012) 073044, doi:10.1088/1367-2630/14/7/073044.

[21] Santos S., Verdaguer A., Souier T., Thomson N. H. and ChiEsA M., Nanotechnology, 22 (2011) 465705.

[22] Yaminsky V. V., Colloids Surf. A, 159 (1999) 181.

[23] IsRaelachvili J., Intermolecular \& Surface Forces (Academic Press) 1991.

[24] Willett C. D., Adams M. J., Johnson S. A. and SEville J. P. K., Langmuir, 16 (2000) 9396.

[25] Derjaguin B. V., Muller V. and Toporov Y., J. Colloid Interface Sci., 53 (1975) 314.

[26] Fischer-CRIPPS A. C., Nanoindentation (Springer, New York) 2004.

[27] Santos S., Guang L., Souier T., Gadelrab K. R., Chiesa M. and Thomson N. H., Rev. Sci. Instrum., 83 (2012) 043707.

[28] Hutter J. L. and Bechhoefer J., Rev. Sci. Instrum., 64 (1993) 1868.

[29] Kawai S., Glatzel T., Koch S., Such B., Baratoff A. and Meyer E., Phys. Rev. B, 80 (2009) 085422. 